\title{
Um einen Tiger zu schlagen brauchst du eines Bruders Hilfe*
}

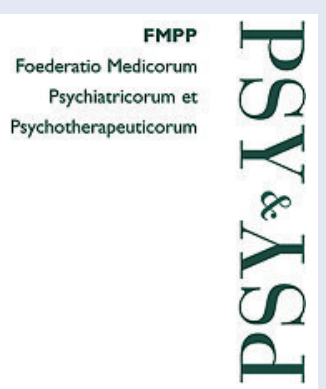

Dr. med. Hans Kurt, Präsident FMPP

* Chinesisches Sprichwort; selbstverständlich sind auch Tigerinnen, Schwestern und Ärztinnen gemeint.

Korrespondenz: Sekretariat SGPP Postgasse 17 Postfach 686 CH-3000 Bern 8 Tel. 0313138833 Fax 0313138899
Vor gut 50 Jahren hat sich die Schweizerische Gesellschaft für Kinder- und Jugendpsychiatrie und Psychotherapie SGKJJP von der Schweizerischen Gesellschaft für Psychiatrie und Psychotherapie SGPP abgetrennt. Seit sieben Jahren haben die beiden Fachgesellschaften wieder enger zueinander gefunden, nämlich im Rahmen des Dachverbandes FMPP, der Verbindung der psychiatrisch-psychotherapeutischen Ärztinnen und Ärzte der Schweiz. Bei Neugründungen von medizinischen Vereinigungen bleibt oft die Wahl zwischen der Tradition, nämlich dem edlen Latein: Foederatio Medicorum Psychiatricorum et Psychotherapeuticorum oder aber dem modernen Englisch, wie etwa Swiss Federation of ...

\section{Unterwegs zur schlank strukturierten Dachgesellschaft}

Aber die gut 2300 FMPP-Miglieder sind nicht Traditionalisten. Der Dachverband der psychiatrischen Fachgesellschaften versucht, gemeinsame Themen zu bearbeiten, d. h. vor allem die standespolitischen, «gewerkschaftlichen» Anliegen und die Öffentlichkeitsarbeit. Gleichzeitig sollen die einzelnen Fachgesellschaften aber ihre Autonomie behalten, wie etwa bei der Weiter- und Fortbildung, der Qualitätssicherung und weiteren fachbezogenen Themen. Kurz gesagt übernimmt die FMPP eher die standes- und gesundheitspolitischen Themen, die einzelnen Fachgesellschaften hingegen den eigenen «Fachbereich». Zur Zeit befindet sich die FMPP in einem Organisationsentwicklungsprozess, die Zusammenarbeit zwischen der SGPP und der SGKJPP soll noch enger werden, die Strukturen müssen schlanker und einfacher werden, und die FMPP als Dachverband soll eine stärkere, demokratisch durch die Mitglieder abgesicherte Legitimation erhalten.

\section{Lobbying für psychisch kranke Menschen}

Die FMPP setzt sich nicht nur für ihre Mitglieder ein, sondern auch für die psychisch kranken Menschen und ihre Angehörigen, handelt es sich doch um eine
Patientengruppe, die über keine Lobby verfügt. Bei den heutigen Sparbemühungen im Gesundheitswesen besteht die Gefahr einer anfänglich impliziten und später schleichend akzeptierten Rationierung. Dies obwohl in den letzten Jahren psychische Leiden zunehmen und eine adäquate und ausreichende Behandlung dieser Erkrankungen mehr denn je nötig ist, nicht zuletzt auch, um die immensen wirtschaftlichen Folgekosten einzuschränken. Die Delegierten der FMPP haben im vergangenen Frühling folgende drei prioritären Anliegen verabschiedet:

- Die Aufhebung des Zulassungsstopps für Fachärzte Psychiatrie und Psychotherapie, gerade auch in Hinsicht auf den Nachwuchsmangel, der die zukünftige Versorgung psychisch kranker Menschen in der Schweiz in Frage stellt (analoge Situation wie bei den Hausärzten).

- In neuen Versorgungsmodellen wie Ärztenetzwerken und Managed Care ist den speziellen Bedürfnissen psychisch kranker Menschen Rechnung zu tragen. Insbesondere muss der direkte, persönliche Zugang zum Psychiater weiterhin gewährleistet bleiben, ist er doch von wesentlicher Bedeutung für den Erfolg einer psychiatrisch-psychotherapeutischen Behandlung.

- Keine Einschränkung der Psychotherapie als wissenschaftlich anerkannter Heilbehandlung in der Grundversicherung, weder mit administrativen Hürden noch bei Langzeitpsychotherapien, dort wo diese indiziert sind.

Die FMPP hat sich in den letzen Jahren in verschiedenen Organen und Aufgaben für die Anliegen einer starken FMH eingesetzt. So ist zu hoffen, dass die Anliegen der FMPP in der gesamten Ärzteschaft Gehör finden, im Sinne einer starken Bruderschaft gegen viele Tiger. 